British Journal of Nutrition (2022), 128, 1758-1770

doi: $10.1017 / \mathrm{S} 0007114521004803$

(C) The Author(s), 2021. Published by Cambridge University Press on behalf of The Nutrition Society. This is an Open Access article, distributed under the terms of the Creative Commons Attribution-NonCommercial-NoDerivatives licence (http://creativecommons.org/licenses/by-nc-nd/4.0/), which permits non-commercial re-use, distribution, and reproduction in any medium, provided that no alterations are made and the original article is properly cited. The written permission of Cambridge University Press must be obtained prior to any commercial use and/or adaptation of the article.

\title{
Effects of L-citrulline supplementation and watermelon consumption on longer-term and postprandial vascular function and cardiometabolic risk markers: a meta-analysis of randomised controlled trials in adults
}

\author{
Ellen T. H. C. Smeets, Ronald P. Mensink and Peter J. Joris* \\ Department of Nutrition and Movement Sciences, NUTRIM School for Nutrition and Translational Research in Metabolism,
} Maastricht University Medical Center, 6200 MD, Maastricht, The Netherlands

(Submitted 28 July 2021 - Final revision received 15 November 2021 - Accepted 30 November 2021 - First published online 6 December 2021 )

Abstract

L-Citrulline may improve non-invasive vascular function and cardiometabolic risk markers through increases in L-arginine bioavailability and nitric oxide synthesis. A meta-analysis of randomised controlled trials (RCT) was performed to examine longer-term and postprandial effects of L-citrulline supplementation and watermelon consumption on these markers for CVD in adults. Summary estimates of weighted mean differences in vascular function and cardiometabolic risk markers with accompanying $95 \%$ CI were calculated using random or fixed-effect meta-analyses. Seventeen RCT were included involving an L-citrulline intervention, of which six studied postprandial and twelve longer-term effects. Five studies investigated longer-term effects of watermelon consumption and five assessed effects during the postprandial phase. Longer-term L-citrulline supplementation improved brachial artery flow-mediated vasodilation (FMD) by $0.9 \%$-point (95\% CI $0 \cdot 7,1 \cdot 1$, $P<0.001)$. Longer-term watermelon consumption improved pulse wave velocity by $0.9 \mathrm{~m} / \mathrm{s}(95 \% \mathrm{CI} 0.1,1.5, P<0.001)$, while effects on FMD were not studied. No postprandial effects on vascular function markers were found. Postprandial glucose concentrations decreased by $0.6 \mathrm{mmol} / \mathrm{l}(95 \% \mathrm{CI} 0 \cdot 4,0 \cdot 7, P<0.001)$ following watermelon consumption, but no other longer-term or postprandial effects were observed on cardiometabolic risk markers. To conclude, longer-term L-citrulline supplementation and watermelon consumption may improve vascular function, suggesting a potential mechanism by which increased $\mathrm{L}$-citrulline intake beneficially affects cardiovascular health outcomes in adults. No effects on postprandial vascular function markers were found, while more research is needed to investigate the effects of L-citrulline and watermelon on risk markers related to cardiometabolic health.

Key words: L-citrulline: Watermelon: Vascular function: Cardiometabolic health: Meta-analysis

L-Citrulline - a non-essential amino acid abundantly found in watermelon - is the immediate precursor for the synthesis of L-arginine ${ }^{(1,2)}$. Through increased L-arginine bioavailability, L-citrulline stimulates the synthesis of endogenous nitric oxide $(\mathrm{NO})^{(3)}$ that may exert several beneficial cardiometabolic and vascular health effects ${ }^{(4-6)}$, thereby reducing the risk on developing $\mathrm{CVD}^{(7)}$. A recent review indeed found that L-citrulline supplementation consistently increased both plasma L-arginine and cyclic guanosine monophosphate concentrations, which is an indicator of NO production ${ }^{(8)}$. We already reported in our very recent meta-analysis of human trials that L-arginine improved longer-term (i.e. $3 \mathrm{~d}$ to 6 months) fasting and 2 -h postprandial vascular endothelial function, which was explained by an increased NO bioavailability ${ }^{(9)}$. Remarkably, increasing evidence now indicates that dietary L-citrulline supplementation may be an even more efficient intervention to increase L-arginine and NO bioavailability than supplementation with L-arginine itself ${ }^{(10-12)}$. In fact, dietary L-arginine is - in contrast to L-citrulline - partly metabolised to L-ornithine and urea by the enzyme arginase in the gastrointestinal tract and the liver resulting in lower plasma L-arginine concentrations as compared with dietary L-citrulline that bypasses intestinal and first hepatic metabolism ${ }^{(8,13,14)}$.

Two meta-analyses of human intervention studies have already reported that L-citrulline supplementation decreases blood pressure ${ }^{(15,16)}$. Also, L-arginine intake has previously been shown to lower TAG concentrations ${ }^{(17)}$ and to play an important role in glucose metabolism ${ }^{(18)}$. It is therefore of interest to study if L-citrulline supplementation may also beneficially affect cardiometabolic risk markers, such as total cholesterol, glucose and

Abbreviations: FMD, flow-mediated vasodilation; NO, nitric oxide; PWA, pulse wave analysis; PWV, pulse wave velocity; RCT, randomised controlled trial; WMD, weighted mean differences.

* Corresponding author: Peter J. Joris, email p.joris@maastrichtuniversity.nl 
insulin concentrations. Studies on these cardiometabolic risk markers are, however, equivocal. In addition, several trials already investigated the effects of dietary L-citrulline on various vascular function markers addressing different aspects of the vasculature during the fasting and postprandial phase, but effects have not been systematically and quantitatively reviewed. Moreover, results of watermelon consumption on vascular function and cardiometabolic risk markers have not been quantitatively summarised before. Therefore, the aim was to investigate and compare the effects of L-citrulline supplementation and watermelon consumption on vascular function and cardiometabolic risk markers in randomised controlled trials (RCT) involving adults. Focus was on longer-term and postprandial effects, while different vascular function markers were considered that exist along the pathway between diet andCVD.

\section{Methods}

\section{Eligibility criteria}

Human RCT investigating postprandial or longer-term effects of L-citrulline supplementation or watermelon consumption with an appropriate control group on (i) non-invasive vascular function parameters and (ii) cardiometabolic risk markers were included. Vascular function parameters included brachial artery flow-mediated vasodilation (FMD), pulse wave analysis (PWA), pulse wave velocity (PWV), and related outcomes. Cardiometabolic risk markers included circulating glucose, serum insulin and total cholesterol concentrations. Only articles published in English involving an adult study population were included. Longer-term was defined as supplementing L-citrulline or consuming watermelon for at least one day.

\section{Search method and data extraction}

Two systematic searches using the electronic databases PubMed, EMBASE, MEDLINE and the Cochrane Library were performed to retrieve potentially relevant articles published before June 2021. A search was conducted for studies assessing effects on: (i) the vascular function parameters and, (ii) cardiometabolic risk markers. The following search terms were used: flow-mediated dilation (or vasodilatation or vasodilation or dilatation or FMD), endothelial (or endothelium) function (or dysfunction), vascular reactivity, PWA, PWV, L-citrulline (or citrulline malate), watermelon (or citrullus lanatus), glucose, insulin (or proinsulin) and insulin resistance (or clamp or glucose tolerance test or matsuda or HOMA-IR or QUICKI). Detailed information on the search terms used can be found in Supplemental Table 1.

Titles and abstracts of all potentially eligible articles were first screened independently by two of the authors. The full text of selected articles was then read. Discrepancies were resolved by discussion until consensus was reached. Studies were excluded if relevant data were missing. Also, studies were not included if the intervention involved infusion of L-citrulline or multiple consecutive bolus ingestions for studies investigating postprandial effects. After inclusion, data were extracted relating to participant (i.e. age, BMI, sex and health status), treatment characteristics (i.e. watermelon or L-citrulline intervention, study duration, relevant outcome parameters with accompanying measures of variability), the total number of dropouts and funding received. If the amount of L-citrulline was not provided, it was calculated using the information that $1000 \mathrm{~g}$ of fresh watermelon flesh corresponds on average to $2 \mathrm{~g}$ of L-citrulline ${ }^{(19)}$. It should be noted, however, that fresh watermelon flesh may contain slightly more L-citrulline than watermelon rind ${ }^{(19)}$. If available, data on circulating markers of L-arginine metabolism (i.e. L-citrulline, L-arginine, L-ornithine an intermediate amino acid involved in the conversion of L-arginine back into L-citrulline ${ }^{(20)}$ - and NOx (i.e. nitrate and nitrite) concentrations) were also extracted.

\section{Risk of bias and GRADE assessment}

Studies were assessed for their risk of bias (i.e. low, some concerns or high risk) using the Cochrane Risk of Bias 2 (RoB 2) assessment tool for randomised parallel and cross-over trials ${ }^{(21)}$. In addition, the quality of evidence (i.e. very low, low, moderate or high) was determined by the GRADE assessment. RCT were qualified as high-quality studies by using this assessment tool, whereas downgraded RCT provided moderate quality of evidence. Finally, (very) low quality of evidence was derived from double or triple-downgraded $\mathrm{RCT}^{(22)}$.

\section{Statistical analyses}

Quantitative meta-analyses were conducted when at least two independent studies investigated the impact of L-citrulline supplementation or watermelon consumption on one of the vascular function parameters or cardiometabolic risk markers. All statistical analyses were performed using Stata 12.1 (Stata Corporation, College Station). As described ${ }^{(23)}$, effect sizes for all outcomes in cross-over trials were calculated by subtracting the mean value at the end of the treatment period from the mean value at the end of the control period. Mean changes in the control group were subtracted from mean changes in the intervention group for outcomes in parallel studies. Mean changes were defined as the difference between the end and start of the study values. Multiple study arms were included if results were only provided for different study populations separately (i.e. men $v$. women, or young $v$. old participants). For postprandial studies, the time point with the most pronounced effect was taken if multiple time points were measured.

For all outcomes, summary estimates of weighted mean differences (WMD) and 95\% CI were determined using fixed-effect meta-analyses and visualised using forest plots. The inverse of the within-subject variance (i.e. 1/(standard error $)^{2}$ ) was used as weighing factor. If only one study was available, no weighting was involved in the calculation of the statistical summary. Heterogeneity was quantified using $\mathrm{I}^{2}$, that is, the percentage of variability in effect estimate that is due to heterogeneity rather than sampling error. If relevant heterogeneity between studies was present, as indicated by an $\mathrm{I}^{2}$ above $50 \%$, random-effect meta-analyses were performed. 

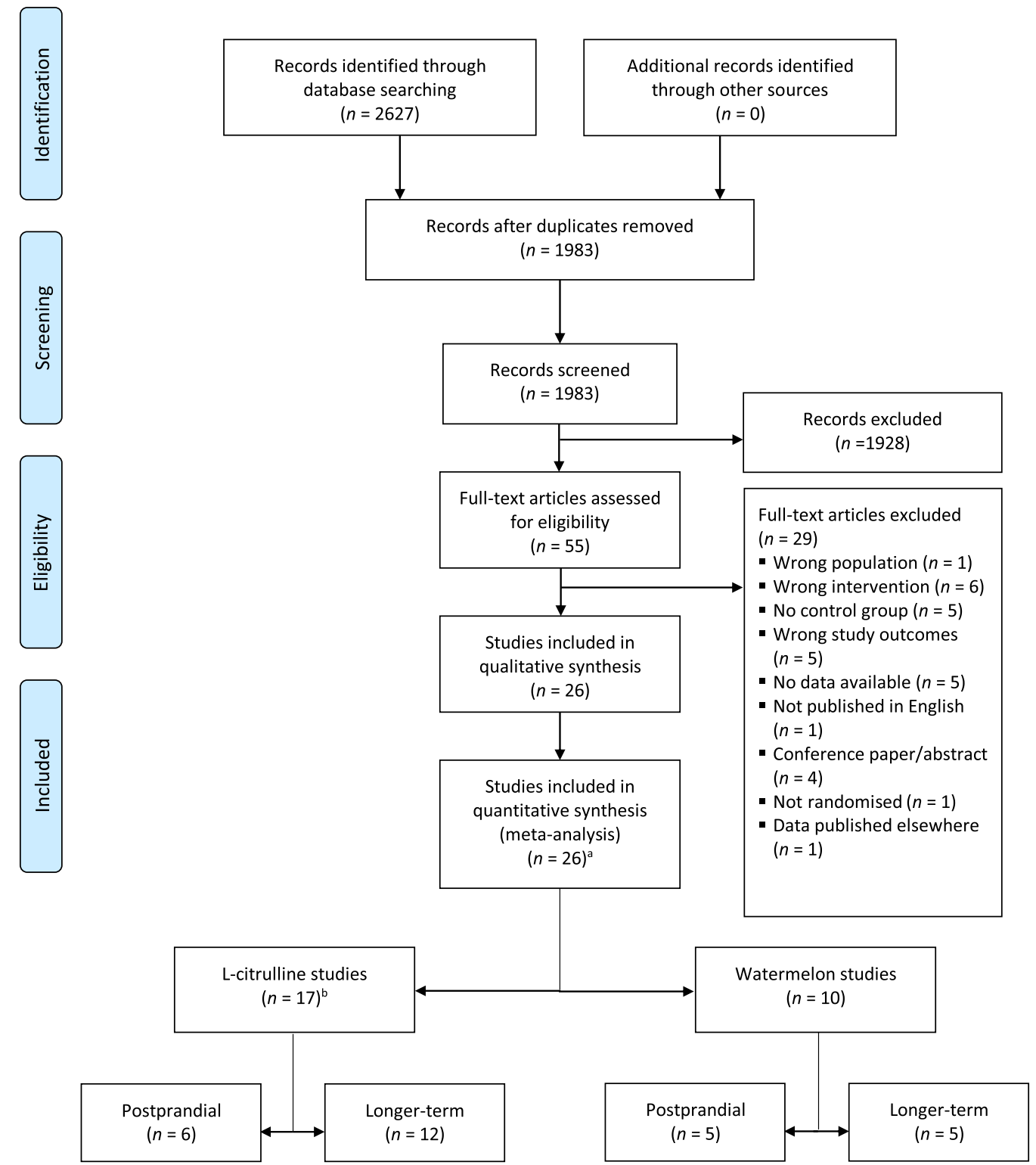

Fig. 1. PRISMA 2009 flow diagram of the study selection process of human randomised controlled trials that investigated postprandial and longer-term effects of $L-$ citrulline supplementation or watermelon consumption on vascular function and cardiometabolic risk markers. ${ }^{a}$ one study included a L-citrulline and watermelon intervention; ${ }^{b}$ one study examined both postprandial and longer-term effects.

\section{Results}

\section{Study selection}

A total of 1983 potentially relevant articles were retrieved from both systematic searches and 1928 papers were excluded for different reasons, such as no inclusion of a watermelon or Lcitrulline intervention, no adult study population or no RCT. The full text of the fifty-five remaining articles were reviewed and twenty-six were included in the present meta-analysis (Fig. 1).

As shown in Fig. 1, seventeen studies involved L-citrulline supplementation. Six studies with eight relevant study arms investigated postprandial effects ${ }^{(24-29)}$, while twelve studies with twelve relevant study arms investigated longer-term effects ${ }^{(27,30-40)}$. One trial investigated both postprandial and longer-term effects ${ }^{(27)}$.
Ten studies involved a watermelon intervention: five studies with five relevant study arms examined postprandial effects ${ }^{(25,41-44)}$ and the other five studies with five relevant study arms investigated longer-term effects of watermelon consumption ${ }^{(45-49)}$. One study included both a $\mathrm{L}$-citrulline and watermelon intervention ${ }^{(25)}$.

\section{Study characteristics}

Three studies did not receive any funding ${ }^{(30,36,40)}$, while one study did not report any information on funding or grants ${ }^{(27)}$. Ten studies mentioned that their supplements were provided by Pronat Laboratories, NOW Foods, Milne Fruits or Cobell Ltd, without receiving any direct funding ${ }^{(31,32,35,37,39,45-49)}$, while two studies reported that they received funding from the National Watermelon Promotion Board ${ }^{(41,44)}$. 
Studies investigating longer-term effects of L-citrulline supplementation had a study duration between 1 week and 4 months ${ }^{(27,30-40)}$. In total, 311 participants were included ranging from fifteen to thirty-eight participants per study arm. Their mean age varied between 22 to 71 years, and their BMI from 23.7 to $35.0 \mathrm{~kg} / \mathrm{m}^{2}$. The dose of L-citrulline ranged from 3 to $6 \mathrm{~g}$ per $\mathrm{d}$. One study provided a dose of $100 \mathrm{mg} / \mathrm{kg}$ per $\mathrm{d}^{(37)}$. Five studies included a healthy study population ${ }^{(27,33,34,37,38)}$. The other studies included participants with overweight and/or obesity ${ }^{(31,32,39)}$, heart failure $^{(30,35)}$, coronary artery disease ${ }^{(36)}$ or type 2 diabetes $^{(40)}$. Postprandial effects were studied after 1 to $5 \mathrm{~h}^{(24-29)}$. These studies included in total of 123 participants ranging from 7 to 27 per study arm. Their mean age was between 21 to 81 years, and their BMI from 23.5 to $29.0 \mathrm{~kg} / \mathrm{m}^{2}$. Studies provided 5.6 to $10 \mathrm{~g}$ of L-citrulline. Six studies involved a healthy study population ${ }^{(24-29)}$ and one study included participants with heart failure ${ }^{(26)}$ (Tables 1 and 2).

Five studies investigated longer-term consumption of a watermelon intervention ${ }^{(45-49)}$. Study duration varied from $16 \mathrm{~d}$ to 6 weeks. These studies included fifty-six participants with a mean age from 22 to 58 years and a mean BMI from 23.6 to $38.1 \mathrm{~kg} / \mathrm{m}^{2}$. One study supplemented with watermelon juice providing $3.4 \mathrm{~g}$ of $\mathrm{L}$-citrulline per $\mathrm{d}^{(45)}$. The remaining studies used watermelon powders or extracts providing 2.7 to $6 \mathrm{~g}$ of $\mathrm{L}$-citrulline per $\mathrm{d}$. Two studies included healthy participants ${ }^{(45,48)}$. The other studies included patients with pre-hypertension ${ }^{(46)}$ or obese patients with (pre)hypertension ${ }^{(47,49)}$. Five studies investigated postprandial effects of watermelon consumption ${ }^{(25,41-44)}$. In total, eighty-six participants ranging from six to twenty-seven per study arm were included. Mean age ranged from 21 to 35 years and mean BMI from 21.2 to $28.7 \mathrm{~kg} / \mathrm{m}^{2}$. Varying amounts of (L-citrullineenriched) watermelon juice ${ }^{(25,41,42)}$ or watermelon ${ }^{(43,44)}$ were provided in the studies corresponding to 0.8 to $6.9 \mathrm{~g}$ of $\mathrm{L}$-citrulline. Four studies involved a healthy study population ${ }^{(25,41-43)}$, whereas one study included overweight and obese participants ${ }^{(44)}$ (see Tables 3 and 4).

\section{Longer-term and postprandial effects on vascular function} markers

Longer-term (i.e. 1 week to 4 months) L-citrulline supplementation significantly improved FMD by $0.9 \%$-point (95\% CI 0.7, 1.1, $P<0.001$, Table 5 and Fig. 2), while no heterogeneity was observed between studies $(P=0 \cdot 85)$. No studies have investigated longer-term effects of watermelon consumption on FMD. Longer-term (i.e. 16 d-6 weeks) watermelon consumption significantly improved PWV by $0.9 \mathrm{~m} / \mathrm{s}$ (95\% CI $-1.5,-0 \cdot 1$, $P<0.001$ ), while L-citrulline supplementation did just not reach statistical significance (WMD $-0.2 \mathrm{~m} / \mathrm{s}, 95 \% \mathrm{CI}-0 \cdot 4,0 \cdot 0$, $P=0 \cdot 10$, Table 5 and Fig. 3). Several PWA-related outcomes (i.e. augmentation index (corrected for heart rate), mean arterial pressure, transit time to reflected wave and augmentation index after a cold pressure test) were assessed in the longer-term trials. No effects of L-citrulline and watermelon were observed on the augmentation index (WMD 0.8\%-point, 95\% CI -2.4, 3.9, $P=0.63$ and WMD $-0.1 \%$-point, 95\% CI -6.9, 6.7, $P=0.99$, respectively). After a cold pressure test, however, L-citrulline supplementation decreased the augmentation index by $7.8 \%$ point (95\% CI $-13 \cdot 0,-2 \cdot 6, P=0 \cdot 003$, Table 5$)$.
Postprandial FMD was not affected by L-citrulline supplementation (WMD $0.1 \%$-point, $95 \% \mathrm{CI}-1 \cdot 9,2 \cdot 2, P=0.89$ ) or watermelon consumption (WMD $0.4 \%$-point, $95 \%$ CI $-1.5,2.3$, $P=0 \cdot 67)$. Only one study investigated postprandial effects of L-citrulline supplementation on PWV and found no significant effect (WMD $-0 \cdot 3 \mathrm{~m} / \mathrm{s}, 95 \% \mathrm{CI}-1 \cdot 1,0 \cdot 4, P=0 \cdot 41$, Table 6). No postprandial studies investigated the effects of L-citrulline or watermelon on augmentation index and other PWA-related outcomes parameters.

\section{Longer-term and postprandial effects on cardiometabolic risk markers}

In only one study, longer-term effects on circulating glucose concentrations after watermelon consumption were reported, but no effects were observed (WMD $0.0 \mathrm{mmol} / \mathrm{l}, 95 \% \mathrm{CI}-0.0$, $0 \cdot 0, P=1 \cdot 00$ ). However, a trend towards reduced glucose concentrations was observed after L-citrulline supplementation (WMD $-0.9 \mathrm{mmol} / 1,95 \% \mathrm{CI}-1.9,0 \cdot 8, P=0 \cdot 06$ ). Total cholesterol concentrations were not changed after longer-term L-citrulline supplementation (WMD $0.0 \mathrm{mmol} / \mathrm{l}, 95 \% \mathrm{CI}-0.4,0.4$, $P=0 \cdot 98$, Table 7).

Postprandial glucose concentrations significantly decreased by $0.6 \mathrm{mmol} / \mathrm{l}$ after watermelon consumption (95\% CI -0.7 , $-0.4, P<0.001)$, with significant heterogeneity between studies $(P<0.001)$. Effects of $\mathrm{L}$-citrulline supplementation on postprandial glucose, insulin and total cholesterol concentrations were only studied in one trial and no effects were found. Moreover, only one trial included postprandial total cholesterol concentrations after watermelon consumption, which were not affected (see Table 8).

\section{Longer-term and postprandial effects on circulating markers of L-arginine metabolism}

Longer-term L-citrulline supplementation and watermelon consumption increased L-arginine concentrations (WMD $40 \cdot 7 \mu \mathrm{mol} / \mathrm{l}$, $95 \%$ CI 19.1, 62.3, $P<0.001$ and WMD $49 \cdot 0 \mu \mathrm{mol} / 1,95 \%$ CI 43.5 , $54.5, P<0.001$, respectively). Longer-term watermelon consumption (WMD $79.0 \mu \mathrm{mol} / 1,95 \%$ CI 66.1, 91.7, $P<0 \cdot 001$ ), but not L-citrulline supplementation $(28.0 \mu \mathrm{mol} / \mathrm{l}, 95 \%$ CI $-15 \cdot 7$, $71 \cdot 7, P=0 \cdot 21$ ), also showed significantly increased L-citrulline concentrations in one study. L-Citrulline and watermelon did not affect NOx concentrations (WMD $-1.6 \mu \mathrm{mol} / 1,95 \% \mathrm{CI}-5.9$, $2 \cdot 7, P=0.46$ and WMD $8.5 \mu \mathrm{mol} / 1,95 \% \mathrm{CI}-78.9,95.9$, $P=0 \cdot 85$, respectively, Table 6). Effects on L-ornithine concentrations were not studied.

L-citrulline supplementation increased significantly postprandial L-citrulline, L-arginine and L-ornithine concentrations (WMD 1647.5 $\mu \mathrm{mol} / 1,95 \%$ CI 1362.7, 1932.1, $P<0 \cdot 001$, WMD $101.7 \mu \mathrm{mol} / 1,95 \%$ CI 87.7 to $115.8, P<0.001$ and WMD $55.8 \mu \mathrm{mol} / 1,95 \%$ CI 35.2, 76.3, $P<0.001$, respectively). Postprandial L-arginine and L-citrulline concentrations were also significantly increased by watermelon consumption (WMD $27.9 \mu \mathrm{mol} / 1,95 \%$ CI 17.7 to $38.1, \quad P<0.001$ and WMD $49.5 \mu \mathrm{mol} / 1,95 \%$ CI 21.9, 77.2, $P<0.001$, see Table 8), while effects on L-ornithine concentrations were not investigated. Only one study examined the effects of L-citrulline supplementation on postprandial NOx concentrations and found no 
Table 1. Overview of studies involving longer-term L-citrulline supplementation on vascular function and cardiometabolic risk markers included in this meta-analysis

\begin{tabular}{|c|c|c|c|c|c|c|c|c|c|c|c|c|c|c|}
\hline $\begin{array}{l}\text { Author and } \\
\text { year }\end{array}$ & $\begin{array}{c}\text { No of par- } \\
\text { ticipants } \\
\text { analysed }\end{array}$ & $\begin{array}{l}\text { No of } \\
\text { dropout }\end{array}$ & $\begin{array}{l}\text { Sex: male } \\
(\%)\end{array}$ & Age (years) & $\begin{array}{c}\mathrm{BMI}(\mathrm{kg} / \\
\left.\mathrm{m}^{2}\right)\end{array}$ & Health status & Treatment & Control & Duration & Amount & $\begin{array}{l}\text { Study } \\
\text { design }\end{array}$ & Funding & $\begin{array}{c}\text { Vascular function } \\
\text { markers }^{\star}\end{array}$ & $\begin{array}{c}\text { Cardiometabolic } \\
\text { risk markers }\end{array}$ \\
\hline \multicolumn{15}{|c|}{ Longer-term studies } \\
\hline Azizi & 45 & 9 & $61 / 68$ & $47 \cdot 6 / 50 \cdot 0$ & $29 \cdot 8 / 28 \cdot 3$ & $\begin{array}{l}\text { Type } 2 \text { diabetes } \\
\text { patients }\end{array}$ & L-citrulline & $\begin{array}{l}\text { Microcrystalline cellu- } \\
\text { lose }\end{array}$ & 8 weeks & $3 \mathrm{~g} / \mathrm{d}$ & Parallel & No funding & & $\begin{array}{l}\text { Glucose, choles- } \\
\text { terol }\end{array}$ \\
\hline $\begin{array}{l}\text { Balderas- } \\
\text { Munoz } \\
\text { 2012(30) }\end{array}$ & 35 & 5 & $80 / 53 \cdot 3$ & $68 \cdot 2 / 65 \cdot 8$ & - & $\begin{array}{l}\text { Heart failure } \\
\text { patients } \\
\text { (NYHA class } \\
\text { I and II) }\end{array}$ & $\begin{array}{l}\text { Citrulline } \\
\text { malate }\end{array}$ & $\begin{array}{l}\text { Optimal pharmaco- } \\
\text { logical treatment }\end{array}$ & 4 months & $3 \mathrm{~g} / \mathrm{d}(2 \times 1.5 \mathrm{~g})$ & Parallel & No funding & Photoplethysmography & \\
\hline $\begin{array}{l}\text { Figueroa } \\
\text { 2010(33) }\end{array}$ & 17 & 1 & 100 & 22 & 25.7 & Healthy & L-citrulline & Maltodextrin & 4 weeks & $6 \mathrm{~g} / \mathrm{d}$ & $\begin{array}{l}\text { Cross- } \\
\text { over }\end{array}$ & $\begin{array}{l}\text { College of Human Sciences } \\
\text { Research Award and } \\
\text { Bess Ward Honors } \\
\text { Thesis award. } \\
\text { Supplements provided } \\
\text { by NOW Foods }\end{array}$ & PWA & \\
\hline $\begin{array}{l}\text { Figueroa } \\
\text { 2015(32) }\end{array}$ & 27 & 0 & $0 / 0$ & $58 / 58$ & $33.8 / 35.0$ & $\begin{array}{l}\text { Obese postme- } \\
\text { nopausal }\end{array}$ & $\begin{array}{l}\text { Whole } \\
\text { body } \\
\text { vobration } \\
\text { exercise } \\
\text { training } \\
+ \text {-cit- } \\
\text { rulline }\end{array}$ & $\begin{array}{c}\text { Whole body vibration } \\
\text { exercise training }+ \\
\text { maltodextrin }\end{array}$ & 8 weeks & $6 \mathrm{~g} / \mathrm{d}(2 \times 3.0 \mathrm{~g})$ & Parallel & $\begin{array}{l}\text { Supplements provided by } \\
\text { NOW Foods }\end{array}$ & PWA, PWV & \\
\hline $\begin{array}{l}\text { Figueroa } \\
2016(31)\end{array}$ & 16 & 0 & 100 & 24.0 & $29 \cdot 3$ & $\begin{array}{l}\text { Overweight and } \\
\text { obese }\end{array}$ & L-citrulline & Maltodextrin & 2 weeks & $6 \mathrm{~g} / \mathrm{d}(2 \times 3.0 \mathrm{~g})$ & $\begin{array}{l}\text { Cross- } \\
\text { over }\end{array}$ & $\begin{array}{l}\text { Supplements provided by } \\
\text { NOW Foods }\end{array}$ & PWA, PWV & \\
\hline $\begin{array}{l}\text { Gonzales } \\
2017(34)\end{array}$ & 25 & 0 & 48 & 70.5 & 23.7 & Healthy & L-Citrulline & Maltodextrin & 2 weeks & $6 \mathrm{~g} / \mathrm{d}(2 \times 3.0 \mathrm{~g})$ & $\begin{array}{l}\text { Cross- } \\
\text { over }\end{array}$ & $\begin{array}{l}\text { American Heart Association } \\
\text { Beginning Grant-in-Aid }\end{array}$ & PWV & \\
\hline $\begin{array}{l}\text { Ochiai } \\
\text { 2012(27) }\end{array}$ & 15 & 0 & 100 & $58 \cdot 5 / 58.0$ & $25 \cdot 2 / 24 \cdot 9$ & Healthy & L-Citrulline & Placebo (unspecified) & 1 week & $5.6 \mathrm{~g} / \mathrm{d}$ & Parallel & Not reported & $\mathrm{PWV} \dagger$ & $\begin{array}{l}\text { Glucose, choles- } \\
\text { terol }\end{array}$ \\
\hline $\begin{array}{c}\text { Orea-Tejeda } \\
2010(35)\end{array}$ & 27 & 3 & $60 \cdot 0 / 53 \cdot 3$ & $66 \cdot 8 / 63 \cdot 1$ & - & $\begin{array}{c}\text { Heart failure } \\
\text { patients } \\
\text { (NYHA class } \\
\text { I and II) }\end{array}$ & $\begin{array}{r}\text { L-citrulline } \\
\text { malate }\end{array}$ & L-arginine $(8 \mathrm{~g} / \mathrm{d})$ & 2 months & $3 \mathrm{~g} / \mathrm{d}$ & Parallel & $\begin{array}{l}\text { Supplements provided by } \\
\text { Pronat Laboratories }\end{array}$ & Photoplethysmography & \\
\hline Safi 2017(36) & 30 & 0 & 60 & 56.5 & - & $\begin{array}{l}\text { Coronary artery } \\
\text { disease } \\
\text { patients }\end{array}$ & L-citrulline & Placebo (unspecified) & $15 \mathrm{~d}$ & $3 \mathrm{~g} / \mathrm{d}$ & $\begin{array}{l}\text { Cross- } \\
\text { over }\end{array}$ & No funding & FMD & \\
\hline $\begin{array}{l}\text { Sanchez- } \\
\text { Gonzalez } \\
\text { 2013(37) }\end{array}$ & 16 & 0 & 100 & 23.0 & $27 \cdot 1$ & Healthy & L-citrulline & Maltodextrin & 2 weeks & $100 \mathrm{mg} /(\mathrm{kg} \times \mathrm{d})$ & $\begin{array}{l}\text { Cross- } \\
\text { over }\end{array}$ & $\begin{array}{l}\text { Supplements provided by } \\
\text { NOW Foods }\end{array}$ & PWA & \\
\hline $\begin{array}{l}\text { Schwedhelm } \\
\text { 2008(38) }\end{array}$ & 20 & 0 & 65.0 & $57 \cdot 0$ & $25 \cdot 6$ & Healthy & L-citrulline & Placebo (unspecified) & 1 week & $6 \mathrm{~g} / \mathrm{d}(2 \times 3.0 \mathrm{~g})$ & $\begin{array}{l}\text { Cross- } \\
\text { over }\end{array}$ & $\begin{array}{l}\text { Angiogenix Inc, Burlingame, } \\
\text { CA, USA and } \\
\text { Angsagenix, Inc }\end{array}$ & FMD & \\
\hline $\begin{array}{l}\text { Wong } \\
\text { 2016(39) }\end{array}$ & 38 & 3 & $0 / 0$ & $58 \cdot 0 / 58 \cdot 0$ & $33 \cdot 8 / 35 \cdot 0$ & $\begin{array}{l}\text { Obese postme- } \\
\text { nopausal }\end{array}$ & $\begin{array}{l}\text { Whole } \\
\text { body } \\
\text { vibration } \\
\text { exercise } \\
\text { training } \\
+ \text { +-cit- } \\
\text { rulline }\end{array}$ & $\begin{array}{c}\text { Whole body vibration } \\
\text { exercise training }+ \\
\text { maltodextrin }\end{array}$ & 8 weeks & $6 \mathrm{~g} / \mathrm{d}(2 \times 3.0 \mathrm{~g})$ & Parallel & $\begin{array}{l}\text { Supplements provided by } \\
\text { NOW Foods }\end{array}$ & PWA & \\
\hline
\end{tabular}

*PWA, pulse wave analysis; PWV, pulse wave velocity; FMD, flow-mediated vasodilation.

†PWV provided as $\mathrm{cm} / \mathrm{s}$ instead of $\mathrm{m} / \mathrm{s}$. Values are converted into $\mathrm{m} / \mathrm{s}$ before analyses. 
NS British Journal of Nutrition

Table 2. Overview of studies involving postprandial L-citrulline supplementation on vascular function and cardiometabolic risk markers included in this meta-analysis

\begin{tabular}{|c|c|c|c|c|c|c|c|c|c|c|c|c|c|c|}
\hline $\begin{array}{l}\text { Author and } \\
\text { year }\end{array}$ & $\begin{array}{l}\text { No of par- } \\
\text { ticipants } \\
\text { analysed }\end{array}$ & $\begin{array}{l}\text { No of } \\
\text { dropout }\end{array}$ & $\begin{array}{c}\text { Gender } \\
\text { Male } \\
(\%) \\
\end{array}$ & $\begin{array}{c}\text { Age } \\
\text { (years) }\end{array}$ & $\begin{array}{c}\mathrm{BMI}(\mathrm{kg} / \\
\left.\mathrm{m}^{2}\right)\end{array}$ & $\begin{array}{l}\text { Health } \\
\text { status }\end{array}$ & Treatment & Control & Duration & Amount & $\begin{array}{c}\text { Study } \\
\text { design }\end{array}$ & Funding & $\begin{array}{c}\text { Vascular } \\
\text { function } \\
\text { markers† }\end{array}$ & $\begin{array}{c}\text { Cardiometabolic } \\
\text { risk markers } \\
\end{array}$ \\
\hline \multicolumn{15}{|c|}{ Postprandial studies } \\
\hline $\begin{array}{l}\text { Churchward- } \\
\text { Venne } \\
2014(24)\end{array}$ & 21 & 0 & 100 & $73.7 / 71.9$ & $28 \cdot 0 / 26 \cdot 7$ & Healthy & $\begin{array}{l}\text { Whey + resis- } \\
\text { tance exer- } \\
\text { cise training } \\
+ \text { L-citrul- } \\
\text { line }\end{array}$ & $\begin{array}{l}\text { Whey + resist- } \\
\text { ance exer- } \\
\text { cise } \\
\text { training }+\mathrm{N}- \\
\text { EAA }\end{array}$ & $5 \mathrm{~h}$ & $10 \mathrm{~g}$ & Parallel & $\begin{array}{l}\text { Canadian } \\
\text { Institutes for } \\
\text { Health } \\
\text { Research and } \\
\text { Natural } \\
\text { Sciences and } \\
\text { Engineering } \\
\text { Research } \\
\text { Council of } \\
\text { Canada } \\
\text { Postgraduate } \\
\text { Scholarship }\end{array}$ & & Glucose, insulin \\
\hline $\begin{array}{l}\text { Cutrufello } \\
2015(25)\end{array}$ & 22 & 0 & 50 & 20.8 & $23 \cdot 5^{*}$ & Healthy & $\begin{array}{l}710 \mathrm{ml} \\
\text { sucrose } \\
\text { solution }+ \\
\text { L-citrulline }\end{array}$ & $\begin{array}{l}710 \mathrm{ml} \text { sucrose } \\
\text { solution }\end{array}$ & $1 \mathrm{~h}$ & $6 \mathrm{~g}$ & Cross-over & Not reported & FMD & \\
\hline $\begin{array}{l}\text { Kim 2015- } \\
\mathrm{a}(26)\end{array}$ & 7 & 0 & 71.4 & 26 & $25 \cdot 6$ & Healthy & L-Citrulline & $\begin{array}{l}\text { Serine, glycine } \\
\text { and alanine }\end{array}$ & $6 \mathrm{~h}$ & $10 \mathrm{~g}$ & Cross-over & $\begin{array}{l}\text { Pepper Center } \\
\text { Grant }\end{array}$ & $\begin{array}{l}\text { Forearm } \\
\text { blood flow }\end{array}$ & \\
\hline $\begin{array}{l}\text { Kim 2015- } \\
\text { b(26) }\end{array}$ & 7 & 0 & 28.6 & 81 & 29.0 & $\begin{array}{l}\text { Heart fail- } \\
\text { ure }\end{array}$ & L-Citrulline & $\begin{array}{l}\text { Serine, glycine } \\
\text { and alanine }\end{array}$ & $6 \mathrm{~h}$ & $10 \mathrm{~g}$ & Cross-over & $\begin{array}{l}\text { Pepper Center } \\
\text { Grant }\end{array}$ & $\begin{array}{l}\text { Forearm } \\
\text { blood flow }\end{array}$ & \\
\hline $\begin{array}{l}\text { Ochiai } \\
\text { 2012(27) }\end{array}$ & 15 & 0 & 100 & $58 \cdot 5 / 58 \cdot 0$ & $25 \cdot 2 / 24 \cdot 9$ & Healthy & L-Citrulline & $\begin{array}{l}\text { Placebo } \\
\quad \text { (unspecified) }\end{array}$ & $1 \mathrm{~h}$ & $5.6 \mathrm{~g}$ & Parallel & Not reported & PWV $\ddagger$ & $\begin{array}{c}\text { Glucose, choles- } \\
\text { terol }\end{array}$ \\
\hline $\begin{array}{l}\text { Rogers } \\
\text { 2020(28) }\end{array}$ & 24 & 3 & 71.4 & $22 \cdot 3$ & $24 \cdot 0^{*}$ & Healthy & L-Citrulline & Dextrose & $1 \mathrm{~h}$ & $8 \mathrm{~g}$ & Cross-over & $\begin{array}{l}\text { State } \\
\text { Undergraduat- } \\
\text { e Research } \\
\text { Fund, } \\
\text { University of } \\
\text { Arkansas } \\
\text { Honors } \\
\text { College } \\
\text { Undergraduat- } \\
\text { e Research } \\
\text { Grant, and } \\
\text { Nutrition 21, } \\
\text { LLC }\end{array}$ & FMD & \\
\hline $\begin{array}{l}\text { Trexler } \\
\text { 2020(29) }\end{array}$ & 27 & 3 & 100 & 22 & 24.8 & Healthy & $\begin{array}{l}\text { L-Citrulline } \\
\text { malate }\end{array}$ & $\begin{array}{l}\text { Blackcurrant } \\
\text { juice }\end{array}$ & $2 \mathrm{~h}$ & $8 \mathrm{~g}$ & Cross-over & $\begin{array}{l}\text { National } \\
\text { Strength and } \\
\text { Conditioning } \\
\text { Association } \\
\text { Foundation }\end{array}$ & $\begin{array}{l}\text { Femoral } \\
\text { artery } \\
\text { blood flow }\end{array}$ & \\
\hline
\end{tabular}

*BMI calculated by provided height and weight

† FMD: flow-mediated vasodilation, PWV: pulse wave velocity.

$\ddagger P W V$ provided as $\mathrm{cm} / \mathrm{s}$ instead of $\mathrm{m} / \mathrm{s}$. Values are converted into $\mathrm{m} / \mathrm{s}$ before analyses. 
Table 3. Overview of the studies investigating postprandial watermelon consumption on vascular function and cardiometabolic risk markers included in this meta-analysis

\begin{tabular}{|c|c|c|c|c|c|c|c|c|c|c|c|c|c|c|c|}
\hline $\begin{array}{l}\text { Author and } \\
\text { year }\end{array}$ & $\begin{array}{c}\text { No of } \\
\text { participants } \\
\text { analysed }\end{array}$ & $\begin{array}{l}\text { No of } \\
\text { dropout }\end{array}$ & $\begin{array}{c}\text { Sex } \\
\text { Male } \\
(\%)\end{array}$ & $\begin{array}{c}\text { Age } \\
\text { (years) }\end{array}$ & $\begin{array}{l}\text { BMl } \\
(\mathrm{kg} / \\
\left.\mathrm{m}^{2}\right)\end{array}$ & $\begin{array}{l}\text { Health } \\
\text { status }\end{array}$ & Treatment & Control & Duration & Amount & Citrulline & $\begin{array}{l}\text { Study } \\
\text { design }\end{array}$ & Funding & $\begin{array}{l}\text { Vascular } \\
\text { function } \\
\text { markers† }\end{array}$ & $\begin{array}{l}\text { Cardiometabolic } \\
\text { risk markers }\end{array}$ \\
\hline \multicolumn{16}{|c|}{ Postprandial studies } \\
\hline $\begin{array}{l}\text { Blohm } \\
\text { 2020(41) }\end{array}$ & 27 & 7 & $48 \cdot 1$ & $24 \cdot 6$ & $22 \cdot 8$ & Healthy & $\begin{array}{l}\text { Watermelon } \\
\text { juice }\end{array}$ & $\begin{array}{l}\text { Gatorade } \\
\text { juice }\end{array}$ & $45 \mathrm{~min}$ & $355 \mathrm{ml}$ & $0.8 \mathrm{~g}$ & Cross-over & $\begin{array}{l}\text { National Watermelon } \\
\text { Promotion Board }\end{array}$ & & Glucose \\
\hline $\begin{array}{l}\text { Cutrufello } \\
2015(25)\end{array}$ & 22 & 0 & 50 & $20 \cdot 8$ & $23 \cdot 5^{*}$ & Healthy & $\begin{array}{l}\text { Watermelon } \\
\text { juice }\end{array}$ & $\begin{array}{l}\text { Sucrose sol- } \\
\text { ution }\end{array}$ & $1 \mathrm{~h}$ & $710 \mathrm{ml}$ & $1.0 \mathrm{~g}$ & Cross-over & Not reported & FMD & \\
\hline $\begin{array}{l}\text { Fan } \\
2020(44)\end{array}$ & 6 & 1 & 33.3 & $32 \cdot 2$ & $28 \cdot 7$ & $\begin{array}{l}\text { Overweight } \\
\text { and } \\
\text { obese }\end{array}$ & $\begin{array}{l}\text { Watermelon } \\
\text { (flesh) }\end{array}$ & Control meal & $8 \mathrm{~h}$ & $278 \mathrm{~g} \ddagger$ & $0.01 \mathrm{~g}$ & Cross-over & $\begin{array}{l}\text { National Watermelon } \\
\text { Promotion Board }\end{array}$ & FMD & \\
\hline $\begin{array}{l}\text { Martinez- } \\
\text { Sanchez } \\
\text { 2017(42) }\end{array}$ & 21 & 0 & 100 & $35 \cdot 3$ & $23 \cdot 9$ & Healthy & $\begin{array}{l}\text { L-Citrulline } \\
\text { enriched } \\
\text { water- } \\
\text { melon } \\
\text { juice }\end{array}$ & $\begin{array}{l}\text { Placebo } \\
\text { juice } \\
\text { (unspeci- } \\
\text { fied) }\end{array}$ & $72 \mathrm{~h}$ & $500 \mathrm{ml}$ & $6.9 \mathrm{~g}$ & Cross-over & $\begin{array}{l}\text { Juan de la Cierva } \\
\text { Grant }\end{array}$ & & $\begin{array}{l}\text { Glucose, choles- } \\
\text { terol }\end{array}$ \\
\hline $\begin{array}{l}\text { Robert } \\
\text { 2008(43) }\end{array}$ & 10 & 0 & 50 & 31.4 & $21 \cdot 2$ & Healthy & Watermelon & Glucose & $2 \mathrm{~h}$ & $893 \mathrm{~g}$ & $1.8 \mathrm{~g} \S$ & Cross-over & $\begin{array}{l}\text { Funding from Universiti } \\
\text { Sains Malaysia, } \\
\text { Cargill Inc., ILSI } \\
\text { Europe, Dutch } \\
\text { Sugar Bureau, and } \\
\text { Mars Inc. }\end{array}$ & & Glucose \\
\hline
\end{tabular}

*BMI calculated by provided height and weight.

† FMD, flow-mediated vasodilation.

Dased on the Dutch nutritional centre indicating that $278 \mathrm{~g}$ of watermelon contain $100 \mathrm{kcal}$

$\S$ Amount of $\mathrm{L}-$ Citrulline not provided but calculated with the information that $1000 \mathrm{~g}$ of watermelon contains approximately $2 \mathrm{~g}$ of $\mathrm{L}$-Citrulline. 
effects (WMD $0 \cdot 1 \mu \mathrm{mol} / 1,95 \% \mathrm{CI}-76 \cdot 8,77 \cdot 1, P=1 \cdot 00$ ). These effects were not investigated after watermelon consumption.

\section{Risk of bias and GRADE assessment}

Longer-term and postprandial L-citrulline studies were all classified as having a low risk of bias. Moreover, the included watermelon studies also had a low risk of bias (online Supplemental Tables $2 \mathrm{a}$ and $\mathrm{b}$ ). GRADE assessment revealed that the included studies provided moderate or high quality of evidence.

\section{Discussion}

In this meta-analysis of human RCT, we found that longer-term Lcitrulline supplementation significantly improved FMD, while watermelon consumption beneficially affected PWV. No postprandial effects of L-citrulline or watermelon on vascular function markers were observed. Watermelon consumption beneficially affected glucose concentrations during the postprandial phase, but other postprandial or longer-term effects on cardiometabolic risk markers were not observed.

Longer-term L-citrulline supplementation significantly improved vascular endothelial function as FMD increased by $0.9 \%$-point. Moreover, we here also provide evidence for an improved arterial stiffness as PWV decreased by $0.9 \mathrm{~m} / \mathrm{s}$ after longer-term watermelon consumption. In line with this, beneficial effects on PWA outcomes were also found after both L-citrulline supplementation and watermelon consumption. These improvements in vascular function are of clinical relevance as they are associated with a reduced risk to develop cardiovascular events $^{(50-53)}$. Longer-term L-citrulline supplementation nearly improved PWV, but effects were less pronounced as compared with those of watermelon. The amount of L-citrulline was comparable between the L-citrulline $(2 \cdot 7-6 \mathrm{~g} / \mathrm{d})$ and watermelon (3$6 \mathrm{~g} / \mathrm{d}$ ) studies, but L-citrulline studies were shorter (i.e. 1 to 4 weeks $v$. 6 weeks), which could be a possible explanation for the observed smaller effects. Moreover, the effects of L-citrulline could have been more pronounced following supplementation with higher dosages were provided, as dose-dependent effects were observed with a maximum effect reached at $10 \mathrm{~g} / \mathrm{d}^{(10)}$. Besides, differential effects between $\mathrm{L}$-citrulline and watermelon on vascular function may also be explained by beneficial effects of other components next to L-citrulline provided watermelon, such as $\mathrm{L}$-arginine, lycopene and vitamin $\mathrm{C}^{(54)}$. In fact, metaanalyses have shown that these components beneficially impact vascular endothelial function as well ${ }^{(55,56)}$ and may also reduce arterial stiffness ${ }^{(57,58)}$.

Since we spend most of the time in the postprandial phase, it is of interest to investigate if watermelon consumption and L-citrulline supplementation also affect vascular function during the postprandial phase. No effects on vascular function markers were found during the postprandial phase. However, most studies assessed vascular function $1 \mathrm{~h}$ postprandially. It cannot be excluded that effects on FMD have not been reached within $1 \mathrm{~h}$ as Borucki and colleagues ${ }^{(59)}$ have indeed shown that 2 -h and 3-h postprandial FMD, but not 1-h FMD, were improved after supplementation with L-arginine. 
Table 5. Effects of longer-term L-Citrulline supplementation and watermelon consumption on vascular function markers

\begin{tabular}{|c|c|c|c|c|}
\hline Variable & No of study arms & WMD & $95 \% \mathrm{Cl}$ & $P$ \\
\hline \multicolumn{5}{|c|}{ Vascular function markers* } \\
\hline \multicolumn{5}{|c|}{ Brachial artery flow-mediated vasodilation (\%) } \\
\hline L-Citrulline & 2 & 0.9 & $0 \cdot 7,1 \cdot 1$ & $<0.001$ \\
\hline Watermelon & - & - & - & - \\
\hline \multicolumn{5}{|c|}{ Pulse wave analysis-related outcomes } \\
\hline \multicolumn{5}{|c|}{ Augmentation index $(\%)$} \\
\hline L-Citrulline & 4 & 0.8 & $-2.4,3.9$ & 0.63 \\
\hline Watermelon & 4 & -0.1 & $-6 \cdot 9,6 \cdot 7$ & 0.99 \\
\hline \multicolumn{5}{|c|}{ Augmentation index corrected for heart rate (Alx75, \%) } \\
\hline L-Citrulline† & 1 & $-4 \cdot 0$ & $-8 \cdot 3,0.3$ & 0.068 \\
\hline Watermelon & 3 & $3 \cdot 3$ & $-4 \cdot 3,11 \cdot 0$ & 0.39 \\
\hline \multicolumn{5}{|c|}{ Mean arterial pressure $(\mathrm{mmHg})$} \\
\hline L-Citrulline & 3 & -0.4 & $-6 \cdot 7,5 \cdot 9$ & 0.91 \\
\hline Watermelon† & 1 & $-6 \cdot 0$ & $-10 \cdot 7,-1 \cdot 3$ & 0.013 \\
\hline \multicolumn{5}{|c|}{ Transit time to reflected wave (ms) } \\
\hline L-Citrulline & 4 & $1 \cdot 0$ & $-3.6,5.5$ & 0.68 \\
\hline Watermelon & 3 & $-5 \cdot 7$ & $-11 \cdot 1,-0.4$ & 0.035 \\
\hline \multicolumn{5}{|c|}{ Augmentation index after cold pressure test (\%) } \\
\hline L-Citrulline & 2 & $-7 \cdot 8$ & $-13 \cdot 0,-2 \cdot 6$ & 0.003 \\
\hline Watermelon† & 1 & $-2 \cdot 1$ & $-5 \cdot 5,1.3$ & 0.23 \\
\hline \multicolumn{5}{|c|}{ Pulse wave velocity $(\mathrm{m} / \mathrm{s})$} \\
\hline L-Citrulline & 5 & -0.2 & $-0.4,0.0$ & 0.096 \\
\hline Watermelon & 2 & -0.9 & $-1 \cdot 3,-0 \cdot 4$ & $<0.001$ \\
\hline \multicolumn{5}{|c|}{ Photoplethysmography (MAT/TT, \%)‡ } \\
\hline L-Citrulline & 2 & $-20 \cdot 1$ & $-56 \cdot 2,15 \cdot 9$ & 0.27 \\
\hline Watermelon & - & - & - & - \\
\hline
\end{tabular}

* No data available for forearm and femoral artery blood flow.

$\dagger n=1$; no weighting is involved in calculation of statistical summary of a single study.

† MAT/TT, maximum amplitude time/total time.

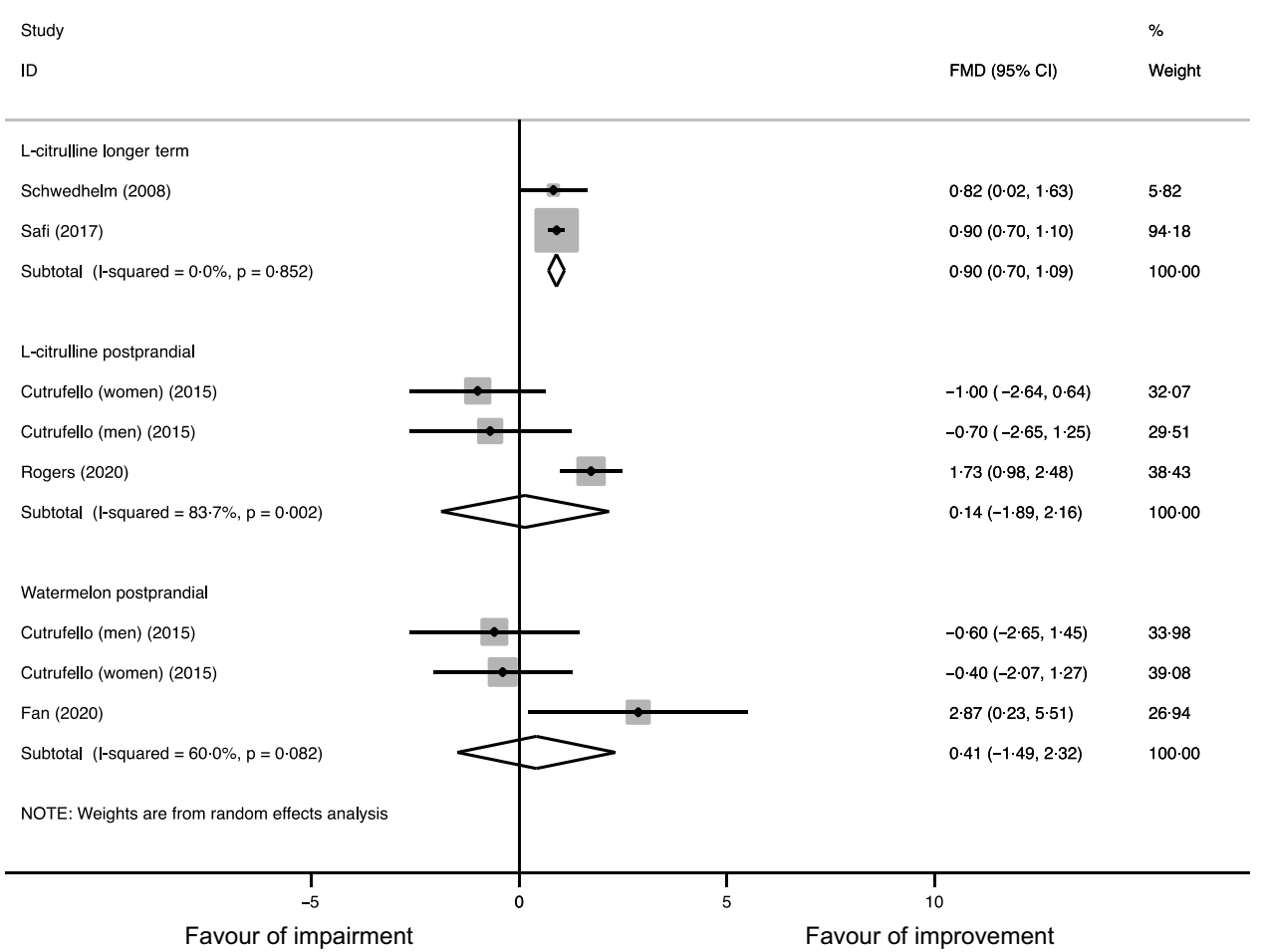

Fig. 2. Forest plot of human randomised controlled trials that investigated postprandial and longer-term effects of L-citrulline supplementation or watermelon consumption on brachial artery flow-mediated vasodilation (FMD). Solid squares represent individual studies, whereas the diamond square represents the weighed mean difference (WMD) in FMD as calculated by random-effect meta-analyses. 


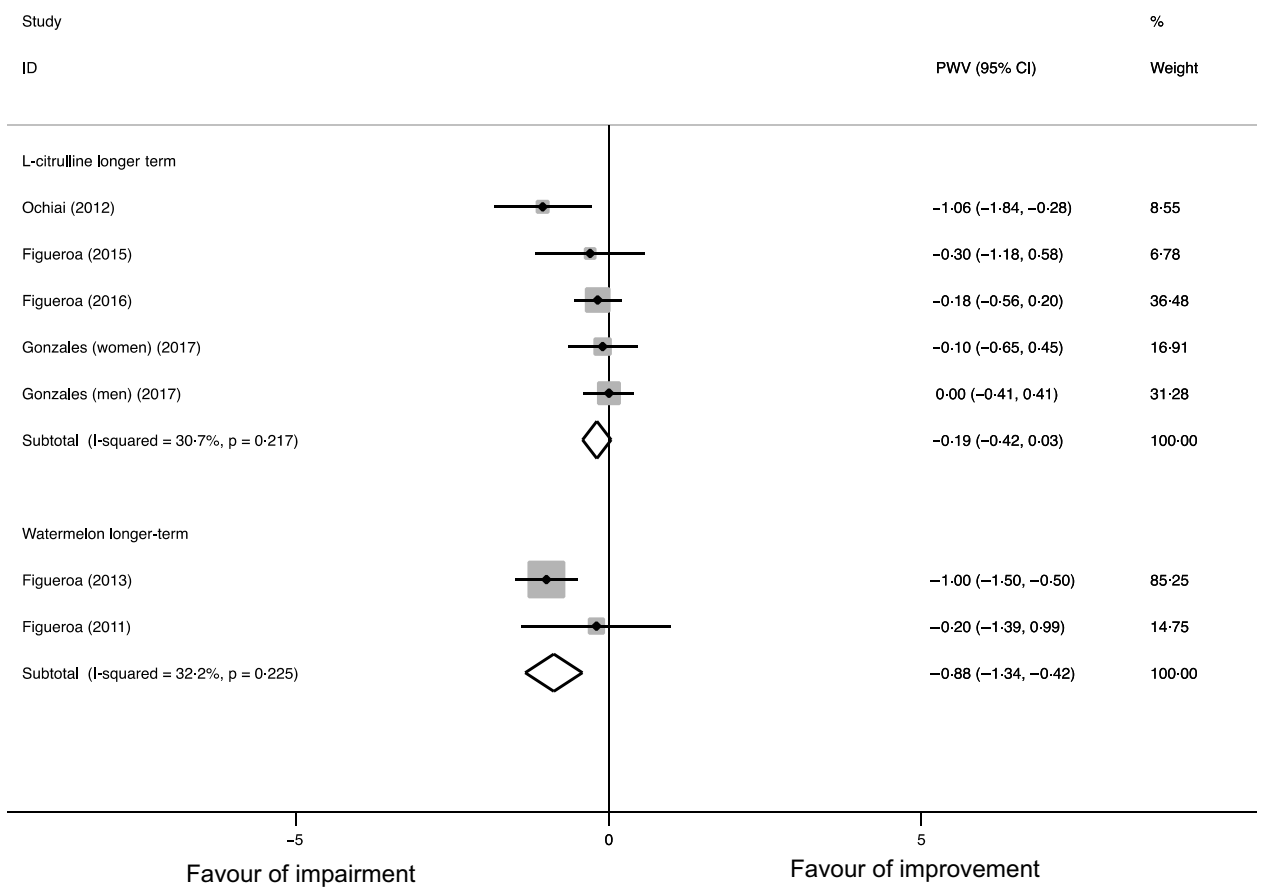

Fig. 3. Forest plot of human randomised controlled trials that investigated postprandial and longer-term effects of $\mathrm{L}$-citrulline supplementation watermelon consumption on pulse wave velocity (PWV). Solid squares represent individual studies, whereas the diamond square represents the weighed mean difference (WMD) in PWV as calculated by fixed-effect meta-analyses.

Overall, cardiometabolic risk markers were not affected. A possible explanation could be that muscle glucose uptake and insulin sensitivity are improved, while fasting circulating concentrations remain unaffected or that the study duration (i.e. 7-16 d) was not long enough to exert long-term effects on fasting circulating glucose concentrations. However, we did observe a postprandial decrease in plasma glucose of $0.6 \mathrm{mmol} / \mathrm{l}$ after watermelon consumption. One mechanism by which watermelon may affect glucose concentrations is by increasing plasma L-arginine concentrations, which may increase NO bioavailability $^{(8)}$ thereby affecting muscle glucose uptake ${ }^{(60)}$. We indeed observed that the consumption of watermelon increased both plasma L-citrulline and L-arginine concentrations during the postprandial phase, which may in turn affect glucose and insulin metabolism via an increased NO bioavailability. However, no changes in longer-term glucose and insulin concentrations were observed.

L-Citrulline, either via supplementation or watermelon intake ${ }^{(61)}$, is not metabolised by intestinal and hepatic arginases, and released as L-arginine into the systemic circulation after conversion by the kidneys ${ }^{(14)}$. This may account for the observation that dietary L-citrulline increases L-arginine and NO bioavailability more efficient than intake of L-arginine itself ${ }^{(10-12)}$. We did observe in this meta-analysis that intake of L-citrulline and watermelon increased postprandial as well as longer-term L-arginine concentrations. However, no effects on plasma nitrate and nitrite concentrations were observed, which may be used as a marker for NO bioavailability. A better approach to assess NO bioavailability is to assess substrates and endogenous inhibitors of endothelial NO synthase, the enzyme responsible for NO production, such as L-arginine, homo-arginine and asymmetric dimethylarginine concentrations ${ }^{(62,63)}$. Therefore, the observed absence of effects on plasma nitrate and nitrite concentrations should be interpreted with caution as they do not necessarily represent an absence of effect on NO bioavailability.

This is the first meta-analysis to compare the effects of watermelon consumption and L-citrulline supplementation on longer-term and postprandial vascular function and cardiometabolic risk markers. Our results are derived from studies with low risk of bias providing moderate-to-high quality of evidence. The protocol was however not pre-registered to PROSPERO and our systematic literature search did not include a search for grey literature. However, our meta-analysis did follow the AMSTAR 2.0 tool and Cochrane handbook ${ }^{(21,64)}$. Another potential limitation is the small number of longer-term studies, while only a limited number of postprandial studies was included. Subgroup analyses could therefore not be performed to investigate if observed results were depending on subject or treatment characteristics. More well-designed trials are thus still needed to further explore health effects and also the safety of watermelon consumption and L-citrulline supplementation. Unfortunately, the studies included did not report plasma L-citrulline concentrations after supplementation. Moreover, it was not mentioned which part of the watermelon was used, which can be important as differences exist in L-citrulline concentrations between rind and flesh. However, most studies did analyse the amount of L-citrulline in the study product. Finally, certain medications may affect L-citrulline metabolism. Furhter investigation is thus still needed to study whether 
Table 6. Postprandial effects of L-Citrulline supplementation and watermelon consumption on vascular function markers.

\begin{tabular}{lcccc}
\hline Variable & $\begin{array}{c}\text { No of study } \\
\text { arms }\end{array}$ & WMD & $95 \% \mathrm{Cl}$ & $P$ \\
\hline
\end{tabular}

Vascular function markers ${ }^{*}$

Brachial artery flow-mediated

vasodilation (\%)

L-Citrulline

Watermelon

Pulse wave velocity $(\mathrm{m} / \mathrm{s})$

L-Citrulline†

Watermelon

Forearm blood flow $(\mathrm{ml} / 100 \mathrm{ml})$

L-Citrulline

Watermelon

Femoral artery blood flow

$(\mathrm{ml} / \mathrm{min} / 100 \mathrm{ml})$

L-Citrulline†

Watermelon

* No data available for all pulse wave analysis-related outcomes and photoplethysmography.

$\dagger n=1$; no weighting is involved in calculation of statistical summary of a single study.

Table 7. Effects of longer-term L-Citrulline supplementation and watermelon consumption on cardiometabolic risk markers and circulating markers of L-arginine metabolism

\begin{tabular}{lcccc}
\hline Variable & $\begin{array}{c}\text { No of study } \\
\text { arms }\end{array}$ & WMD & $95 \% \mathrm{Cl}$ & $P$ \\
\hline
\end{tabular}

Cardiometabolic risk markers

Glucose (mmol/l)

\begin{tabular}{|c|c|c|c|c|}
\hline L-Citrulline & 2 & -0.9 & $-1 \cdot 9,0.8$ & 0.06 \\
\hline Watermelon† & 1 & 0.0 & $-0.0,0.0$ & 1.00 \\
\hline \multicolumn{5}{|c|}{ Total cholesterol (mmol/l) } \\
\hline L-Citrulline & 2 & 0.0 & $-0.4,0.4$ & 0.98 \\
\hline Watermelon & - & - & - & - \\
\hline \multicolumn{5}{|c|}{$\begin{array}{l}\text { Circulating plasma markers of L-arginine metabolism* } \\
\text { L-Citrulline }(\mu \mathrm{mol} / \mathrm{l})\end{array}$} \\
\hline L-Citrulline† & 1 & $28 \cdot 0$ & $-15 \cdot 7,71 \cdot 7$ & 0.21 \\
\hline Watermelon† & 1 & $79 \cdot 0$ & $66 \cdot 1,91 \cdot 7$ & $<0.00$ \\
\hline \multicolumn{5}{|c|}{ L-Arginine $(\mu \mathrm{mol} / \mathrm{l})$} \\
\hline L-Citrulline & 4 & $40 \cdot 7$ & $19 \cdot 1,62 \cdot 3$ & $<0.0$ \\
\hline Watermelon $†$ & 1 & $49 \cdot 0$ & $43 \cdot 5,54 \cdot 7$ & $<0.00$ \\
\hline \multicolumn{5}{|l|}{$\mathrm{NOx}(\mu \mathrm{mol} / \mathrm{l})$} \\
\hline L-Citrulline & 3 & $-1 \cdot 6$ & $-5.9,2.7$ & 0.46 \\
\hline Watermelon† & 1 & 8.5 & $-78.9,95.9$ & 0.85 \\
\hline
\end{tabular}

WMD, weighted mean differences.

* No data available for insulin and L-ornithine concentrations.

$\dagger n=1$; no weighting is involved in calculation of statistical summary of a single study.

the use of medication affects potential benefits of L-citrulline on CVD risk ${ }^{(65)}$

\section{Conclusion}

In conclusion, this meta-analysis provided evidence that longerterm L-citrulline and watermelon consumption may improve vascular function in adults, suggesting a potential mechanism by which an increased L-citrulline intake may beneficially affect cardiovascular health outcomes. No effects on postprandial vascular function markers were found. Longer-term effects on cardiometabolic risk markers were not observed, but more research is still needed to investigate the effects of $\mathrm{L}$-citrulline and watermelon on cardiometabolic health.
Table 8. Postprandial effects of L-citrulline supplementation and watermelon consumption on cardiometabolic risk markers and circulating markers of L-arginine metabolism

\begin{tabular}{|c|c|c|c|c|}
\hline Variable & $\begin{array}{c}\text { No of study } \\
\text { arms }\end{array}$ & WMD & $95 \% \mathrm{Cl}$ & $P$ \\
\hline \multicolumn{5}{|c|}{ Cardiometabolic risk markers } \\
\hline \multicolumn{5}{|c|}{ Glucose $(\mathrm{mmol} / \mathrm{l})$} \\
\hline L-Citrulline* & 1 & 0.6 & $-0 \cdot 8,2 \cdot 1$ & 0.40 \\
\hline Watermelon & 4 & -0.6 & $-0.7,-0.4$ & $<0.001$ \\
\hline \multicolumn{5}{|l|}{ Insulin $(\mu \mathrm{U} / \mathrm{ml})$} \\
\hline L-Citrulline* & 1 & $-6 \cdot 2$ & $-20 \cdot 7,6 \cdot 4$ & 0.41 \\
\hline Watermelon & - & - & - & - \\
\hline \multicolumn{5}{|c|}{$\begin{array}{l}\text { Total cholesterol } \\
\qquad(\mathrm{mmol} / \mathrm{l})\end{array}$} \\
\hline L-Citrulline* & 1 & 0.0 & $-1.6,1.5$ & 0.97 \\
\hline Watermelon* & 1 & -0.2 & $-0.5,0.2$ & 0.31 \\
\hline \multicolumn{5}{|c|}{$\begin{array}{l}\text { Circulating plasma markers of L-arginine metabolism } \\
\text { L-Citrulline }(\mu \mathrm{mol} / /)\end{array}$} \\
\hline L-Citrulline & 3 & $1647 \cdot 5$ & $1362 \cdot 7,1932 \cdot 1$ & $<0.001$ \\
\hline Watermelon* & 1 & 49.5 & $21 \cdot 9,77 \cdot 2$ & $<0.001$ \\
\hline \multicolumn{5}{|c|}{ L-Arginine $(\mu \mathrm{mol} / \mathrm{l})$} \\
\hline L-Citrulline & 3 & $101 \cdot 7$ & $87 \cdot 7,115 \cdot 8$ & $<0.001$ \\
\hline Watermelon & 2 & $27 \cdot 9$ & $17 \cdot 7,38 \cdot 1$ & $<0.001$ \\
\hline \multicolumn{5}{|c|}{ L-Ornithine $(\mu \mathrm{mol} / \mathrm{l})$} \\
\hline L-Citrulline & 2 & $55 \cdot 8$ & $35 \cdot 2,76 \cdot 3$ & $<0.001$ \\
\hline Watermelon & - & - & - & - \\
\hline \multicolumn{5}{|l|}{$\mathrm{NOx}(\mu \mathrm{mol} / \mathrm{l})$} \\
\hline L-Citrulline* & 1 & 0.1 & $-76 \cdot 8,77 \cdot 1$ & 1.00 \\
\hline Watermelon & - & - & - & - \\
\hline
\end{tabular}

WMD, weighted mean differences.

${ }^{*} n 1$; no weighting is involved in calculation of statistical summary of a single study.

\section{Acknowledgement}

This research did not receive any specific grant from funding agencies in the public, commercial or not-for-profit sectors.

E. S. designed the study, performed the literature search, data extraction and statistical analyses, interpreted the data, and wrote the manuscript; R. P. M. designed the study, interpreted the data and wrote the manuscript; P. J. J. designed the study, performed the literature search, interpreted the data, wrote the manuscript and had overall responsibility.

There are no conflicts of interest.

\section{Supplementary material}

For supplementary material/s referred to in this article, please visit https://doi.org/10.1017/S0007114521004803

\section{References}

1. Davis AR, Webber CL, Fish WW, et al. (2011) L-citrulline levels in watermelon cultigens tested in two environments, Hort. Science 46, 1572-1575.

2. Papadia C, Osowska S, Cynober L, et al. (2018) Citrulline in health and disease. Rev bum Stud Clin Nutr 37, 1823-1828.

3. Boger RH (2014) The pharmacodynamics of L-arginine. Altern Ther Health Med 20, 48-54.

4. Levine AB, Punihaole D \& Levine TB (2012) Characterization of the role of nitric oxide and its clinical applications. Cardiology 122, 55-68.

5. Gheibi S \& Ghasemi A (2020) Insulin secretion: the nitric oxide controversy. EXCLIJ 19, 1227-1245. 
6. Hadi A, Arab A, Moradi S, et al. (2019) The effect of L-arginine supplementation on lipid profile: a systematic review and metaanalysis of randomised controlled trials. Br J Nutr $\mathbf{1 2 2}$ 1021-1032.

7. Bryan NS (2018) Functional nitric oxide nutrition to combat cardiovascular disease. Curr Atheroscler Rep 20, 21.

8. Allerton TD, Proctor DN, Stephens JM, et al. (2018) L-citrulline supplementation: impact on cardiometabolic health. Nutrients 10, 921 .

9. Smeets ETHC, Mensink RP \& Joris PJ (2021) Effects of tree nut and groundnut consumption compared with those of L-arginine supplementation on fasting and postprandial flow-mediated vasodilation: meta-analysis of human randomized controlled trials. Clin Nutr 40, 1699-1710.

10. Moinard C, Nicolis I, Neveux N, et al. (2008) Dose-ranging effects of citrulline administration on plasma amino acids and hormonal patterns in healthy subjects: the Citrudose pharmacokinetic study. Br J Nutr 99, 855-862.

11. Suzuki T, Morita M, Hayashi T, et al. (2017) The effects on plasma L-arginine levels of combined oral L-citrulline and Larginine supplementation in healthy males. Biosci Biotechnol Biochem 81, 372-375.

12. Figueroa A, Wong A, Jaime SJ, et al. (2017) Influence of L-citrulline and watermelon supplementation on vascular function and exercise performance. Curr Opin Clin Nutr Metab Care 20, 92-98.

13. Castillo L, deRojas TC, Chapman TE, et al. (1993) Splanchnic metabolism of dietary arginine in relation to nitric oxide synthesis in normal adult man. Proc Natl Acad Sci USA 90, 193-197.

14. van de Poll MC, Siroen MP, van Leeuwen PA, et al. (2007) Interorgan amino acid exchange in humans: consequences for arginine and citrulline metabolism. Am J Clin Nutr 85, 167-172.

15. Yang HH, Li XL, Zhang WG, et al. (2019) Effect of oral L-citrulline on brachial and aortic blood pressure defined by resting status: evidence from randomized controlled trials. Nutr Metab 16, 89

16. Barkhidarian B, Khorshidi M, Shab-Bidar S, et al. (2019) Effects of L-citrulline supplementation on blood pressure: a systematic review and meta-analysis. Avicenna J Phytomed 9, 10-20.

17. Sepandi M, Abbaszadeh S, Qobady S, et al. (2019) Effect of L-Arginine supplementation on lipid profiles and inflammatory markers: a systematic review and meta-analysis of randomized controlled trials. Pharmacol Res 148, 104407.

18. Hu S, Han M, Rezaei A, et al. (2017) L-arginine modulates glucose and lipid metabolism in obesity and diabetes. Curr Protein Pept Sci 18, 599-608.

19. Rimando AM \& Perkins-Veazie PM (2005) Determination of citrulline in watermelon rind. J Chromatogr A 1078, 196-200.

20. Luiking YC, Engelen MP \& Deutz NE (2010) Regulation of nitric oxide production in health and disease. Curr Opin Clin Nutr Metab Care 13, 97-104.

21. Sterne JAC, Savovic J, Page MJ, et al. (2019) RoB 2: a revised tool for assessing risk of bias in randomised trials. BMJ 366, 14898.

22. Schuneman H, Brozek J, Guyatt G, et al. (2013) Handbook for Grading the Quality of Evidence and the Strength of Recommendations Using the GRADE Approace. GRADE Handbook. https://www.gradepro.org/ (accessed June 2021).

23. Ras RT, Hiemstra H, Lin Y, et al. (2013) Consumption of plant sterol-enriched foods and effects on plasma plant sterol concentrations - a meta-analysis of randomized controlled studies. Atherosclerosis 230, 336-346.

24. Churchward-Venne TA, Cotie LM, MacDonald MJ, et al. (2014) Citrulline does not enhance blood flow, microvascular circulation, or myofibrillar protein synthesis in elderly men at rest or following exercise. Am J Physiol Endocrinol Metab 307, E71-83.

25. Cutrufello PT, Gadomski SJ \& Zavorsky GS (2015) The effect of L-citrulline and watermelon juice supplementation on anaerobic and aerobic exercise performance. J Sports Sci $\mathbf{3 3}$, 1459-1466.

26. Kim IY, Schutzler SE, Schrader A, et al. (2015) Acute ingestion of citrulline stimulates nitric oxide synthesis but does not increase blood flow in healthy young and older adults with heart failure. Am J Physiol Endocrinol Metab 309, E915-24.

27. Ochiai M, Hayashi T, Morita M, et al. (2012) Short-term effects of L-citrulline supplementation on arterial stiffness in middle-aged men. Int J Cardiol 155, 257-261.

28. Rogers JM, Gills J \& Gray M (2020) Acute effects of Nitrosigine $(\mathrm{R})$ and citrulline malate on vasodilation in young adults. J Int Soc Sports Nutr 17, 12.

29. Trexler ET, Keith DS, Lucero AA, et al. (2020) Effects of citrulline malate and beetroot juice supplementation on energy metabolism and blood flow during submaximal resistance exercise. J Diet Suppl 17, 698-717.

30. Balderas-Munoz K, Castillo-Martinez L, Orea-Tejeda A, et al. (2012) Improvement of ventricular function in systolic heart failure patients with oral L-citrulline supplementation. Cardiol J 19, 612-617.

31. Figueroa A, Alvarez-Alvarado S, Jaime SJ, et al. (2016) L-citrulline supplementation attenuates blood pressure, wave reflection and arterial stiffness responses to metaboreflex and cold stress in overweight men. Br J Nutr 116, 279-285.

32. Figueroa A, Alvarez-Alvarado S, Ormsbee MJ, et al. (2015) Impact of L-citrulline supplementation and whole-body vibration training on arterial stiffness and leg muscle function in obese postmenopausal women with high blood pressure. Exp Gerontol 63, 35-40.

33. Figueroa A, Trivino JA, Sanchez-Gonzalez MA, et al. (2010) Oral L-citrulline supplementation attenuates blood pressure response to cold pressor test in young men. Am J Hypertens 23, $12-16$.

34. Gonzales JU, Raymond A, Ashley J, et al. (2017) Does L-citrulline supplementation improve exercise blood flow in older adults? Exp Physiol 102, 1661-1671.

35. Orea-Tejeda A, Orozco-Gutierrez JJ, Castillo-Martinez L, et al. (2010) The effect of L-arginine and citrulline on endothelial function in patients in heart failure with preserved ejection fraction. Cardiol J 17, 464-470.

36. Safi M, Mahjoob MP, Nateghi S, et al. (2017) The assessment of short-term effect of L-citrulline on endothelial function via FMD to NMD ratio in known CAD patients: a randomized, cross-over clinical trial. Rom J Intern Med 55, 23-27.

37. Sanchez-Gonzalez MA, Koutnik AP, Ramirez K, et al. (2013) The effects of short term L-citrulline supplementation on wave reflection responses to cold exposure with concurrent isometric exercise. Am J Hypertens 26, 518-526.

38. Schwedhelm E, Maas R, Freese R, et al. (2008) Pharmacokinetic and pharmacodynamic properties of oral L-citrulline and L-arginine: impact on nitric oxide metabolism. Br J Clin Pharmacol 65, 51-59.

39. Wong A, Alvarez-Alvarado S, Jaime SJ, et al. (2016) Combined whole-body vibration training and L-citrulline supplementation improves pressure wave reflection in obese postmenopausal women. Appl Physiol Nutr Metab 41, 292-297.

40. Azizi S, Mahdavi R, Mobasseri M, et al. (2021) The impact of L-citrulline supplementation on glucose homeostasis, lipid profile, and some inflammatory factors in overweight and obese patients with type 2 diabetes: a double-blind randomized placebo-controlled trial. Phytother Res 35, 3157-3166. 
41. Blohm K, Beidler J, Rosen P, et al. (2020) Effect of acute watermelon juice supplementation on post-submaximal exercise heart rate recovery, blood lactate, blood pressure, blood glucose and muscle soreness in healthy non-athletic men and women. Int J Food Sci Nutr 71, 482-489.

42. Martinez-Sanchez A, Ramos-Campo DJ, Fernandez-Lobato B, et al. (2017) Biochemical, physiological, and performance response of a functional watermelon juice enriched in L-citrulline during a half-marathon race. Food Nutr Res $\mathbf{6 1}$, 1330098.

43. Robert SD, Ismail AA, Winn T, et al. (2008) Glycemic index of common Malaysian fruits. Asia Pac J Clin Nutr 17, 35-39.

44. Fan J, Park E, Zhang L, et al. (2020) Pharmacokinetic parameters of watermelon (rind, flesh, and seeds) bioactive components in human plasma: a pilot study to investigate the relationship to endothelial function. J Agric Food Chem 68, 7393-7403.

45. Bailey SJ, Blackwell JR, Williams E, et al. (2016) Two weeks of watermelon juice supplementation improves nitric oxide bioavailability but not endurance exercise performance in humans. Nitric Oxide 59, 10-20.

46. Figueroa A, Sanchez-Gonzalez MA, Perkins-Veazie PM, et al. (2011) Effects of watermelon supplementation on aortic blood pressure and wave reflection in individuals with prehypertension: a pilot study. Am J Hypertens 24, 40-44.

47. Figueroa A, Sanchez-Gonzalez MA, Wong A, et al. (2012) Watermelon extract supplementation reduces ankle blood pressure and carotid augmentation index in obese adults with prehypertension or hypertension. Am J Hypertens 25, 640-643.

48. Figueroa A, Wong A, Hooshmand S, et al. (2013) Effects of watermelon supplementation on arterial stiffness and wave reflection amplitude in postmenopausal women. Menopause 20, 573-577.

49. Figueroa A, Wong A \& Kalfon R (2014) Effects of watermelon supplementation on aortic hemodynamic responses to the cold pressor test in obese hypertensive adults. Am J Hypertens 27, 899-906.

50. Thijssen DHJ, Bruno RM, van Mil A, et al. (2019) Expert consensus and evidence-based recommendations for the assessment of flow-mediated dilation in humans. Eur Heart $J$ 40, 2534-2547.

51. Ras RT, Streppel MT, Draijer R, et al. (2013) Flow-mediated dilation and cardiovascular risk prediction: a systematic review with meta-analysis. Int J Cardiol 168, 344-351.
52. Munakata M (2014) Brachial-ankle pulse wave velocity in the measurement of arterial stiffness: recent evidence and clinical applications. Curr Hypertens Rev 10, 49-57.

53. Zhong Q, Hu MJ, Cui YJ, et al. (2018) Carotid-femoral pulse wave velocity in the prediction of cardiovascular events and mortality: an updated systematic review and meta-analysis. Angiology 69, 617-629.

54. United States Department of Argiculture Research Service (US ARS) (2015) USDA National Nutrient Database for Standard Reference. Service AR. https://fdc.nal.usda.gov/fdc-app. html\#/food-details/167765/nutrients (accessed May 2021).

55. Cheng HM, Koutsidis G, Lodge JK, et al. (2017) Tomato and lycopene supplementation and cardiovascular risk factors: a systematic review and meta-analysis. Atherosclerosis 257, 100-108.

56. Ashor AW, Siervo M, Lara J, et al. (2015) Effect of vitamin C and vitamin E supplementation on endothelial function: a systematic review and meta-analysis of randomised controlled trials. Br J Nutr 113, 1182-1194.

57. Ashor AW, Siervo M, Lara J, et al. (2014) Antioxidant vitamin supplementation reduces arterial stiffness in adults: a systematic review and meta-analysis of randomized controlled trials. J Nutr 144, 1594-1602.

58. Mozos I, Stoian D, Caraba A, et al. (2018) Lycopene and vascular health. Front Pharmacol 9, 521.

59. Borucki K, Aronica S, Starke I, et al. (2009) Addition of $2.5 \mathrm{~g}$ $\mathrm{L}$-arginine in a fatty meal prevents the lipemia-induced endothelial dysfunction in healthy volunteers. Atherosclerosis 205 , $251-254$.

60. Stamler JS \& Meissner G (2001) Physiology of nitric oxide in skeletal muscle. Physiol Rev 81, 209-237.

61. Assefa AD, Hur OS, Ro NY, et al. (2020) Fruit morphology, citrulline, and arginine levels in diverse watermelon (citrullus lanatus) germplasm collections. Plants $\mathbf{9}, 1054$.

62. Forstermann U \& Sessa WC (2012) Nitric oxide synthases: regulation and function. Eur Heart J 33, 829-837.

63. Winkler MS, Kluge S, Holzmann M, et al. (2017) Markers of nitric oxide are associated with sepsis severity: an observational study. Crit Care 21, 189

64. Shea BJ, Reeves BC, Wells G, et al. (2017) AMSTAR 2: a critical appraisal tool for systematic reviews that include randomised or non-randomised studies of healthcare interventions, or both. BMJ 358, j4008.

65. Irving BA, Carter RE, Soop M, et al. (2015) Effect of insulin sensitizer therapy on amino acids and their metabolites. Metabolism 64, 720-728. 